\title{
Cultura para la transformación vs la mentalidad de superviviente
}

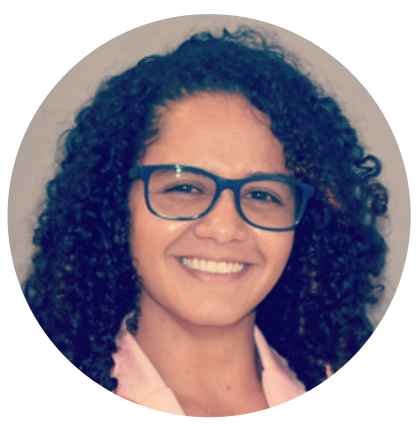

\begin{abstract}
MARCELA GÓMEZ
Coordinadora STEM del Colegio Colombo Hebreo (Colombia). Fundadora del Colectivo Tejido STEM Colombia. Miembro del Colectivo Nacional de Educación STEM - CONASTEM y del Colectivo Latinoamericano Manifiesto STEAM. Miembro asesor de la Mesa Técnica Nacional de Educación STEM+A para el Ministerio de Educación Nacional de Colombia. Ingeniera mecánica y Magíster en ciencias naturales y matemática de la Universidad Pontificia Bolivariana (Colombia). Diplomada en educación STEM/STEAM por la OEA. STEM.org Certified Educational Instructor.
\end{abstract}

marcelagq1987@gmail.com

(0)@marcela.stem

"[...] De la misma manera que los grandes cambios generados por la revolución industrial dieron origen a las nuevas ideologías del siglo XX, es probable que las revoluciones venideras en biotecnología y tecnología de la información requieran perspectivas nuevas" (Harari, 2018, p. 35).

En una variedad importante de escenarios, tanto académicos como coloquiales, el término innovación ha asumido una posición de privilegio que ha trascendido las fronteras de las organizaciones en general. Esto, dada su relación con las transformaciones del entorno y sus circunstancias: los modelos para definir el desarrollo han cambiado, pasando de priorizar el mero crecimiento económico a buscar un desarrollo en conceptos humanos (Max-Neef et al., 1986). Dicho modelo de desarrollo humano ha venido avanzando a su vez para visibilizar adecuadamente criterios como los de equidad y sostenibilidad, cuyos componentes se han revisado y discutido a la luz de marcos de referencia cada vez más globales (ONU, 2015).

Esta perspectiva sistémica del crecimiento ha promovido reflexiones y modelos basados en una nueva cosmovisión del hombre y de su relación con el entorno. Desde el modelo jerárquico, formulado por las Escrituras de distintas religiones como el cristianismo, hasta el modelo sistémico, y en cierto modo animista que rigió en épocas antiguas, la humanidad en general ha estado vinculada de forma compleja a su entorno, tanto el natural como el creado por sus propias manos. La aplicación de estos modelos en la observación de la acción humana cotidiana, ha mostrado una dinámica de retroalimentación que permite apreciar los efectos de ciertas decisiones y acciones del ser humano en las circunstancias del entorno que lo alberga (Norman, 2013) para la definición de más y mejores rutinas de trabajo en pro del bienestar sostenible que se pretende. Como consecuencia de esta perspectiva sistémica, nuevas posibilidades se han hecho evidentes a la vista de más gremios, promoviendo el estudio del impacto que tienen sus procesos y procedimientos, de manera directa e indirecta, en la consecución de sus objetivos colectivos; de estos resultados se desprenden los planes de capacitación y el entrenamiento necesarios para su logro.

Sin duda, las prioridades para el desarrollo han cambiado. En palabras de Oppenheimer (2014, p. 11):

"Hoy en día, la prosperidad de los países depende cada vez menos de sus recursos naturales y cada vez más de sus sistemas educativos y sus innovadores. Los países más exitosos no son los que tienen más petróleo, [...] sino los que desarrollan las mejores mentes y exportan productos con mayor valor agregado".

La democratización de las tecnologías para la transformación digital, como objetivo [1], es parte importante de los análisis y comentarios compartidos por referentes de distintas disciplinas, que van desde la investigación aplicada hasta la historia y la sociología. La hiperconexión permite acceder a un sinnúmero de datos en muy poco tiempo, así como a la interacción remota con dispositivos y sistemas de cada vez mayor sofisticación, y ahora con el refinamiento de diversas tecnologías disruptivas que incluyen a las cuánticas, se abren cada vez más las puertas a la toma de decisiones para la sostenibilidad de diversas iniciativas que pueden mejorar la calidad 
de vida de las comunidades (BID, 2019). E dinamismo creciente de los últimos tiempos ha dejado en claro que la incertidumbre es parte indiscutible del día a día, haciendo que las probabilidades de cambiar la ruta trazada para el sostenimiento de cualquier entidad en el tiempo sean cada vez más altas. Esto, en otras palabras, significa que la innovación va más allá de la simple nominación de estrategias y se asienta en una cultura de y para la transformación.

¿Y en qué consiste la transformación? En la modificación parcial o total de los aspectos que conforman un sistema para alcanzar un mejor desempeño, lo cual se verá determinado por la consistencia entre lo esperado y lo obtenido, así como por la estabilidad de su comportamiento (Mortán Santa Catalina, 2010). Desde esta definición, es posible afirmar que un sistema de comportamiento estable y coherente con el resultado esperado, es el producto de un diseño juicioso previo, seguido de una etapa rigurosa de experimentación. Ambas etapas favorecen la calibración de este sistema mediante una serie de ensayos que permiten ajustar parámetros y detalles menores, en comparación con un proceso improvisado y sin fundamentación, que generalmente es susceptible de reinicio.

¿Qué ocurre en América Latina? ¿Por qué la transformación no es (tan) perceptible a los ojos de la comunidad en general? Si las noticias, las investigaciones, los resultados alcanzados por otros países están al alcance de muchos, ¿por qué tarda tanto el desarrollo? Por una cosmovisión cultural que da mayor relevancia a preconceptos asociados a la mentalidad del superviviente: priorizar los resultados inmediatos que garanticen bienestar (aunque sea momentáneo), disminuyendo al máximo el displacer que trae consigo la toma de decisiones trascendentes, ya que esto puede atribuirse sin problema a un incremento en la peligrosidad del entorno. Estos preconceptos conforman lo que puede llamarse la estructura inercial cultural de una comunidad, es decir, la estructura mental colectiva que condiciona la toma de decisiones mediante la declaración explícita o tácita de los principios que la rigen (Gómez, 2017a, 2017b); para el caso latinoamericano, la estructura inercial cultural se ha anquilosado, manteniéndose en la añoranza de los años 70 y 80 y posponiendo las decisiones que implican mirar hacia épocas que no han venido, pero se adivinan.

Este tipo de constructo cultural, que llamaremos mentalidad, es el que permite establecer una ruta de razonamiento según elementos innegociables para la permanencia de la comunidad; América Latina ha demostrado que su mentalidad está basada en la falsa seguridad, entendiéndose desde el paradigma inmunológico [2] mencionado por Byung Chul-Han (2012, p. 12). ¿De qué otra manera puede explicarse que, de acuerdo con el Banco Mundial (2018), durante el año 2018, la inversión en I+D para varios países de América Latina [3] estuvo entre el $0.03 \%$ y el $0.54 \%$ de su PIB, mientras que países como Estados Unidos, Noruega, Finlandia y Alemania reportaron una inversión superior al $2 \%$ de su PIB durante el mismo año, e Israel invirtió el 4.95\% de su PIB para este propósito? Esto no es todo: el mismo Banco Mundial indica que para el año 2019, estos mismos países latinoamericanos reportaron una inversión de su PIB hasta 10 veces superior a su inversión en educación. ¿Es un error interpretar estas decisiones como consecuencias de una visión pesimista, pasiva e irresponsable del papel que tiene la comunidad latinoamericana en la construcción de su propia realidad? ¿Qué otra cosa, sino el miedo, hace pensar tan evidentemente en el encierro?

Asumiendo que este tipo de realidad huxley-orwelliana no es la esperada, no es descabellado pensar en la modificación de este patrón colectivo [4], la cual debe llevarse a cabo paulatina y disciplinadamente, y para lograr tal cometido, es necesaria la redefinición explícita de sus vectores directores comunitarios, esto es, sus principios. Semejante panorama invita a la consolidación de una mentalidad para el aprendizaje (Gómez, 2019), la cual establezca las bases de una cultura para la innovación (Wagner, 2012) que permita a las dinámicas investigativas, a la ciencia y a la tecnología, soportar el delineamiento de alternativas, el diseño de prototipos y la toma informada de decisiones dentro de todo grupo humano, apelando siempre al carácter influenciador del ser humano y al entrenamiento de su conciencia para la percepción de los posibles futuros que puede construir con sus pares. Al respecto, hay dos aspectos habituales que preocupan por su frecuente manifestación en nuestra idiosincrasia: la impaciencia ante la falta aparente de retroalimentación cuando se ponen a prueba iniciativas de cualquier índole, y la tendencia a la irresponsabilidad individual. Por lo visto, será necesario introducir más que nunca elementos de diseño contextual para que este redireccionamiento de nuestra mentalidad comunitaria pueda verse finalmente a la luz de nuestra esencia cultural y fun- 
damentación histórica; de la conciencia sobre las características únicas de nuestra ubicación geográfica, nuestros recursos naturales, infraestructurales y humanos; de las particularidades demográficas de nuestra comunidad latinoamericana y de sus necesidades en el corto, mediano y largo plazo. Estas perspectivas, espejos de quienes somos, son insumos de real valor para diseñar los caminos que en verdad necesitamos en aras del desarrollo al cual aspiramos.

[1] Si bien ha existido una serie bien conocida de tendencias disruptivas en términos tecnológicos, entre las cuales se incluye la computación cuántica, el tiempo de respuesta de las comunidades emergentes como la latinoamericana, ha sido considerablemente lento. Esto, sumado a la priorización de otros aspectos para la inversión estatal en cada país, acentúa la brecha entre el nivel de desarrollo deseado y las condiciones de inequidad actuales.

[2] Según Chul-Han, el paradigma inmunológico establece una marcada división entre lo de adentro y lo de afuera, lo propio y lo extraño, extendiéndose más allá de lo biológico hasta embeberse incluso en lo social, lo político y lo militar. Se caracteriza por una innegable aversión a lo extraño, independientemente de cuáles sean sus intenciones: se convierte en sujeto de rechazo debido a su otredad.

[3] Se cuentan en esta lista a Argentina, Bolivia, Colombia, Ecuador y Perú. El único país que rompe este patrón de inversión es Brasil, reportando para el mismo período una inversión del $1.26 \%$ de su PIB para I+D (Banco Mundial, 2018).

[4] Concibiendo el comportamiento humano como un sistema de alta complejidad, en el que variables individuales y colectivas tienen un impacto considerable en la respuesta de cada individuo en el corto, mediano y largo plazo.

\section{Referencias}

Banco Interamericano de Desarrollo - BID (2019). Tecnologías cuánticas. Una oportunidad transversal e interdisciplinar para la transformación digital y el impacto social. https://cutt.ly/BkunMCg

Banco Mundial (2018). Gasto en investigación y desarrollo (\% del PIB). https://cutt.ly/hkuROy느

Chul-Han, B. (2012). La sociedad del cansancio. Herder.

Gómez, L. M. (18 de septiembre de 2017a). Educación de calidad y mentalidad de superviviente: la educación no va sólo en el aula. Las notas de clase de Marcela Gómez. https://cutt.ly/Akufdte

Gómez, L. M. (10 de octubre de 2017b). Educación de calidad y mentalidad de superviviente II: la educación y la innovación toman tiempo. Las notas de clase de Marcela Gómez. https://cutt.ly/akukoq!

Gómez, L. M. (2019) Educación STEM/STEAM como pretexto para la innovación en comunidades de aprendizaje. En N. M. Cáceres (Ed.), Educación STEM / STEAM, apuestas hacia la formación, impacto y proyección de seres críticos (p. 56). Fondo Editorial Universitario Servando Garcés de la
Universidad Politécnica Territorial de Falcón.

Harari, Y. N. (2018). 21 lecciones para el siglo XXI. Penguin Random House Grupo Editorial

Max-Neef, M., Elizalde, A., \& Hopenhayn, M. (1986) Desarrollo a Escala Humana, una opción para el futuro. CEPAUR.

Morlán, I. (2010) Modelo de Dinámica de Sistemas para la implantación de Tecnologías de la Información en la Gestión Estratégica Universitaria [Tesis doctoral, Universidad del País Vasco] Repositorio institucional EHU. http://www.ehu.eus/i.morlan/tesis/memoria/TesisIM02.pdf

Norman, D. (2013) The Design of Everyday Things, Revised and Expanded Version. Basic Books.

Oppenheimer, A. (2014) iCrear o morir! La esperanza de América Latina y las cinco claves de la innovación. Penguin Random House Grupo Editorial.

Organización de las Naciones Unidas - ONU (2015). Objetivos de Desarrollo Sostenible. https://cutt.ly/Hkuk2wr

Wagner, T. (2012) Crear innovadores. La formación de los jóvenes que cambiarán el mundo. Norma.

\section{Cómo citar este artículo:}

Gómez, M. (2021). Cultura para la transformación vs la mentalidad de superviviente. Futuro Hoy, 2(1), 13-15. https://doi.org/10.5281/zenodo.4654843

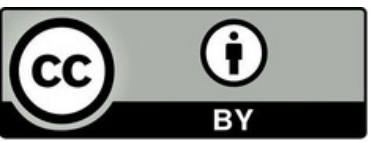

Esta obra está bajo licencia internacional Creative Commons 4.0 Reconocimiento 4.0. 